Revista Destaques Acadêmicos, Lajeado, v. 10, n. 3, 2018. ISSN 2176-3070

DOI: http://dx.doi.org/10.22410/issn.2176-3070.v10i3a2018.1745

http://www.univates.br/revistas

\title{
RELAÇÃO ENTRE CANDIDÍASE DE REPETIÇÃO, DISBIOSE INTESTINAL E SUPLEMENTAÇÃO COM PROBIÓTICOS: UMA REVISÃO
}

\author{
Rafaela Mulinari Paludo ${ }^{1}$, Débora Marin ${ }^{2}$
}

\begin{abstract}
Resumo: Objetivo: Revisar os estudos sobre os efeitos dos probióticos na regulação da microflora intestinal e a possível relação com a melhora do quadro de candidíase e de candidíase de repetição. Método: Tratou-se de um estudo de revisão com abordagem qualitativa. Resultados: A candidíase vaginal é uma infecção fúngica, que afeta a maioria das mulheres em idade fértil. A disbiose é um desequilíbrio da microbiota intestinal que pode afetar o estado de saúde do ser humano, inclusive o sistema imunológico e o trato urogenital das mulheres. Os probióticos são alimentos funcionais, compostos por microrganismos vivos, que atuam na melhora do quadro de disbiose intestinal quando consumidos de forma regular e em quantidade suficiente. Considerações finais: Concluiu-se por meio deste estudo que os probióticos apresentam efeitos benéficos no equilíbrio da flora intestinal, além de serem coadjuvantes no tratamento para candidíase vaginal e também de repetição.
\end{abstract}

Palavras-chave: Candidíase. Candidíase vulvovaginal. Disbiose. Probióticos. Lactobacillus.

\section{INTRODUÇÃO}

A vagina é colonizada por Lactobacillus (cerca de 50\%) e por outras espécies bacterianas e de leveduras como a Candida albicans. Em menor proporção, ela é habitada por Candida glabrata e Candida parapsilosis. Para que os Lactobacillus prevaleçam na flora, é necessário manter ácido o $\mathrm{pH}$ da região (entre 3,5 e 4,5). Estes microrganismos convivem em equilíbrio, podendo tornar-se patógenos em determinadas situações (BALDIM, I.M.; PEREIRA, M.A.; RUFINO, L.R.A.; OLIVEIRA, N.M.S.; FIORINI, J.E., 2012; FARJADO, A.C.S., 2015).

1 Enfermeira. Centro Universitário UNIVATES. Pós-graduada em Enfermagem em Urgências e Emergências. AVM Faculdade Integrada. Enfermeira do Município de Estrela - RS.

2 Nutricionista. Mestre em Biotecnologia pela Universidade do Vale do Taquari - Univates. Docente de cursos de Pós-graduação Lato Sensu da Univates. 
A candidíase vaginal é uma infecção fúngica, que afeta a maioria das mulheres em idade fértil. É caracterizada por corrimento vaginal em grumos semelhantes ao leite qualhado e com prurido intenso. Além dessas características, outros sintomas que podem aparecer são dispareunia, desconforto vulvar e disúria, sem via de regra (BALDIM, I.M.; PEREIRA, M.A.; RUFINO, L.R.A.; OLIVEIRA, N.M.S.; FIORINI, J.E., 2012).

A microbiota intestinal é composta por bactérias, fungos, vírus e elementos genéticos fundamentais à saúde humana. Exerce funções metabólicas, desenvolve o sistema imunológico e atua, quando em equilíbrio, como barreira contra a invasão de patógenos (PEREIRA, I.G.; FERRAZ, I.A.R., 2017). As principais bactérias que compõem a microbiota intestinal não são nocivas à saúde, portanto podem ser consideradas probióticas, como ocorre com as bifidobactérias e com os Lactobacillus (PAIX ÂO, L.A.; CASTRO, F.F.S., 2016).

A disbiose intestinal é um desequilíbrio da microbiota que pode afetar o estado de saúde do ser humano, incluindo o sistema imunológico e o trato urogenital das mulheres (PEREIRA, I.G.; FERRAZ, I.A.R., 2017). Disbioses têm sido verificadas em diversas doenças, dentre as quais podemos destacar os distúrbios vaginais, como a candidíase vaginal (FIORINI, J.E., 2012).

A partir dessas definições, esse trabalho tem por objetivo revisar os estudos sobre os efeitos do uso de probióticos na regulação da microflora intestinal e sua possível relação com a melhora do quadro de candidíase de repetição.

\section{REFERENCIAL TEÓRICO}

\subsection{Candidíase vaginal}

Na vagina, a microbiota é constituída por inúmeros microrganismos que vivem em equilíbrio, dentre os quais se destacam os Lactobacillus, que variam de zero a quatro espécies, podendo ser influenciados por fatores hormonais, métodos contraceptivos, dietas, relação sexual, uso de antibióticos, duchas vaginais, cirurgias ginecológicas e câncer cervical. Em mulheres saudáveis, a população de Lactobacillus é determinada em $10^{7}$ e $10^{8}$ Unidades Formadoras de Colônia por mililitro (UFC/ml) de exsudado vaginal (MARTINEZ, R.C.R., 2008).

A cândida está presente na microbiota vaginal normal e pode ser encontrada na pele, em mucosas e no trato gastrointestinal. Em cultivo in vitro, espécies de cândida apresentam coloração creme, se desenvolvem em condições aeróbicas, em temperatura de $20^{\circ} \mathrm{C}$ e $38^{\circ} \mathrm{C}$ e apresentam $\mathrm{pH}$ entre 2,5-7,5. Quando em desequilíbrio por fatores hormonais, citopatológicos e/ou imunes, prolifera-se desordenadamente, ocasionando a infecção (MELO, B.J.C.; 
NASCIMENTO, E.E.F.; SOARES, S.J.T.; SOUZA, L.T.S.; PARAHYM, A.M.R.C., 2016; MATSUBARA, V.H., 2016).

Candidíase é uma infecção causada por fungos de diversas espécies, sendo a segunda vaginite mais frequente, perdendo apenas pelas vaginoses bacterianas (HOLANDA, A. A. R.; FERNANDES, A. C. S.; BEZERRA, C. M.; FERREIRA, M. A. F.; HOLANDA, M. R. da R.; HOLANDA, J. C. P.; MILAN, E. P., 2007). A cepa mais encontrada nessa infecção fúngica é Candida albicans, que é diagnosticada em cerca de $90 \%$ dos casos. A cepa mais resistente a antifúngicos é a Candida glabrata, responsável por menos de $5 \%$ das infecções (BARBOSA, C.; FERNANDES, G.; QUINTAS, C.; TEIXEIRA, E.M.; NEVES, J.P., 2012).

Em virtude da sua colonização, o tecido cutâneo perianal e o reto apresentam a cândida como principal fonte de infecção. A contaminação a partir do trato digestivo ocorre devido à proximidade entre o ânus e a vagina, podendo desencadear com facilidade episódios de candidíase vaginal, podendo torná-las recorrentes (HOLANDA, A. A. R.; FERNANDES, A. C. S.; BEZERRA, C. M.; FERREIRA, M. A. F.; HOLANDA, M. R. da R.; HOLANDA, J. C. P.; MILAN, E. P., 2007).

Estima-se que $75 \%$ das mulheres em idade fértil apresentarão pelo menos um episódio de candidíase no decorrer da vida adulta, sendo que $5 \%$ delas terão candidíase de repetição por diversos fatores (MELO, B.J.C.; NASCIMENTO, E.E.F.; SOARES, S.J.T.; SOUZA, L.T.S.; PARAHYM, A.M.R.C.,2016). A infecção fúngica de repetição pode afetar a saúde física e emocional do indivíduo, bem como interferir negativamente nos relacionamentos conjugais e sexuais (MARTINEZ, R.C.R., 2008).

Durante a gestação, a suscetibilidade à infecção fúngica é ampliada devido à cândida, por isso, estima-se que de 70 a $85 \%$ das gestantes colonizadas pelo fungo contaminem seus bebês na hora do parto e em torno de 22 a $24 \%$ adquirem Candida albicans oral (MARTINEZ, R.C.R., 2008).

O tratamento geralmente é realizado por derivados azólicos, como o fluconazol, o itraconazol, o cetoconanazol, o sertaconazol e o miconazol, o que apresenta pouca eficácia, pois torna as mulheres resistentes e suas vaginites reincidentes. Por isso, é importante identificar o agente etiológico por meio de cultura antes de iniciar o tratamento, principalmente nos casos de Candida glabrata, quando a infecção apresenta resistência ao fluconazol, mesmo apresentando sintomas diferenciados, como secreção aquosa, ardor intenso e prurido discreto (BARBOSA, C.; FERNANDES, G.; QUINTAS, C.; TEIXEIRA, E.M.; NEVES, J.P., 2012; RODRIGUES, M.T.; GONÇALVES, A.C.; ALVIM, M.C.T.; CASTELLANO FILHO, D.S.; ZIMMERMMANN, J.B.; SILVA, V.L.; DINIZ, C.G., 2013; FEUERSCHUETTE, O.H.M.; SILVEIRA, S.K.; FEUERSCHUETTE, I.; CORRÊA, T.; GRANDO, L.; TREPANI, A., 2010). 
Devido aos casos de infecções recorrentes, terapias alternativas têm sido propostas, promovendo, dessa forma, a utilização de ácido bórico e de probióticos em seu tratamento (CARVALHO, F.P, 2007).

\subsection{Disbiose intestinal}

O intestino é o órgão responsável pela digestão e pela absorção de nutrientes. Além disso, tem função imunológica. Sua mucosa fica exposta diariamente a diversas bactérias e microrganismos invasores, que precisam ser limitados pela barreira intestinal. Para que a função imune do intestino atue corretamente, é necessária a apresentação de três componentes essenciais: a barreira intestinal, o sistema imune e a microflora (FONSECA, F.C.P.; COSTA, C.L., 2010).

A microflora é constituída por diversas bactérias, fungos e microrganismos. Sabe-se que a interação dessas bactérias com o hospedeiro, melhora o equilíbrio da flora intestinal (MATSUBARA, V.H., 2016). O desequilíbrio da microflora (disbiose) é caracterizado pelo predomínio de bactérias patogênicas. A disbiose permite, desse modo, que o intestino fique suscetível a infecções e a desordens imunes. Assim, a nutrição tem papel importante para manter a integridade intestinal, oferecendo nutrientes específicos que melhorem e restabeleçam a flora (FONSECA, F.C.P.; COSTA, C.L., 2010). Disbioses têm sido provocadas por diversas doenças, como a obesidade, a diabetes e os distúrbios vaginais. Com isso, a microflora pode ser manipulada por meio da dieta, que pode melhorar a saúde ou prejudicá-la (FARJADO, A.C.S., 2015).

Quando se fala em microrganismos nocivos presentes no trato gastrointestinal, destaca-se a Candida albicans como fungo mais prevalente, afetando principalmente o esôfago. Ela também está presente no intestino delgado e no intestino grosso, podendo estar associada à úlcera gástrica, retardando a cicatrização, agravando a lesão e destruindo as barreiras fisiológicas normais (MATSUBARA, V.H.; 2016).

Dentre as principais causas de disbiose está o uso irracional de antibióticos, o consumo excessivo de alimentos processados, a excessiva exposição a toxinas ambientais, as disfunções hepatopancreáticas, o estresse, a idade, o tempo de trânsito e o $\mathrm{pH}$ intestinal, a disponibilidade de material fermentável e o estado imunológico do indivíduo (FERREIRA, G.S., 2014).

\subsection{Probióticos}

Os probióticos são alimentos funcionais, compostos por microrganismos vivos, que atuam na melhora do quadro de disbiose intestinal quando consumidos de forma regular e em quantidade adequada (FONSECA, F.C.P.; COSTA, C.L., 2010). 
Para que um probiótico possa ser utilizado na alimentação ou como componente de uma medicação. Ele precisa sobreviver à passagem do trato gastrointestinal e se proliferar no intestino (VANDENPLAS, Y.; HUYS, G; DAUBE G., 2015). Os probióticos são encontrados em alimentos fermentados, como iogurtes e/ou suplementos que contém microrganismos vivos e atuam de forma benéfica no desenvolvimento da microbiota intestinal. Também são conhecidos como bioterapêuticos, bioprotetores e bioprofiláticos, por prevenirem infecções entéricas e gastrointestinais (JACOBY, J.T.; GUZZON, S.; ROESCH, L.F.W.; MENDES, R.H; 2017; MORAES, F.P.; COLLA, L.M, 2006).

No Brasil, podem ser encontrados em formas farmacêuticas (cápsulas ou saches) ou naturais (leite fermentados, iogurtes, queijos, sorvetes e outros alimentos vegetais fermentados), podendo conter apenas um probiótico, ou um conjunto deles (FERREIRA, G.S., 2014). Os probióticos podem ser utilizados como suplementos na dieta, com objetivo de melhorar a saúde, promovendo a melhora das funções naturais do corpo (MATSUBARA, V.H., 2016).

Em um intestino saudável, predominam os microrganismos benéficos e promotores de saúde. Eles pertencem aos gêneros Lactobacillus $(L)$ e Bifidobacterium (B). Como probióticos podemos destacar os seguintes Lactobacillus: L. casei, L. acidophilus, L. delbreuckii subsp. bulgaricus, L. brevis, $L$. cellibiosus, L. lactis, L. fermentum, L. plantarum e L. reuteri. e as bifidobacterias: B. bifidum, B. longum, B. infantis, B. adolescentis, B. thermophilum e B. animalis. Existem ainda algumas bactérias ácidas-lácticas, consideradas probióticos que são as Enteroccocus, faecalis, Enteroccocus faecium e Sporolactobacillus inulinus (MORAES, F.P.; COLLA, L.M., 2006).

Na microflora intestinal, os probióticos atuam na formação de uma barreira protetora, impedindo as bactérias de ligarem-se aos seus receptores, sendo excluídas do meio (FONSECA, F.C.P.; COSTA, C.L., 2010). Atuam também no trato gastrointestinal diminuindo diarreias, aliviando sintomas de intolerância a lactose e de síndrome do intestino irritável, combatem e previnem colite pseudomembranosa e diarreias associadas ao uso de antibióticos (MATSUBARA, V.H.; 2010).

Os probióticos devem, portanto, ser administrados com cautela em indivíduos que fazem uso de varfarina e imunossupressores, como a ciclosporina, a tacrolimus, a azatiprina e osagentes quimioterápicos, pois podem causar infecções ou colonizações (FARJADO, A.C.S., 2015).

\section{METODOLOGIA}

Trata-se de um estudo de revisão com abordagem qualitativa. Esse método propicia a consistência dos resultados para a avaliação do que há de mais relevante nas publicações científicas sobreo tema nos últimos anos.

Para essa revisão buscou-se artigos científicos publicados em revistas, jornais e periódicos, além de dissertações. Os artigos foram pesquisados em 
bases eletrônicas do Scielo, Google Acadêmico e LILACS, usando-se os termos Candida albicans, candidíase de repetição, vulvovaginites, cândida, disbiose intestinal, microflora intestinal e probióticos.

Foram considerados artigos redigidos em português e inglês, publicados entre os anos de 2006 a 2017.

\section{RESULTADOS E DISCUSSÃO}

Quando os probióticos são administrados em quantidades adequadas, oferecem benefícios à saúde, pois têm função de melhorar a barreira imunológica do intestino e são utilizados no tratamento de disbioses (PAIXÃO, L.A.; CASTRO, F.F.S., 2016).

Em um estudo realizado por Baldin et al. (2012) com 44 amostras de secreção vaginal de pacientes e enfermeiras da Clínica de Ginecologia e Obstetrícia do Hospital Universitário Alzira Velano de Alfenas - MG, observouse que o quefir (probiótico apresentado em grão ou grumos, composto por microrganismos como os Lactobacillus e as leveduras que vivem em simbiose) apresentou uma eficácia de $42 \%$ na inibição das leveduras analisadas.

Martinez (2008) afirma que alguns probióticos possuem efeitos benéficos no tratamento de desordens intestinais e auxiliam na prevenção e no tratamento de doenças no trato urogenital. Em estudos realizados com humanos, observou-se elevação da produção e da atividade de fagócitos, aumento sistêmico das respostas de imunoglobulinas A na mucosa intestinal e cancelamento da produção de linfócitos e de citocinas inflamatórias por células T., tornando o trato vaginal menos suscetível á colonização de patógenos.

Fonseca e Costa (2010) relataram um estudo experimental com ratos, o qual foi realizado por Shen e colaboradores (2006). Nesse estudo foi investigada a influência da nutrição enteral, parenteral e probiótica no sistema imune intestinal. Concluiu-se que a administração de nutrição enteral com probióticos melhora a barreira intestinal, inibindo o crescimento de patógenos, equilibrando a flora e aumentando o sistema imune do intestino, reduzindo, portanto, a translocação bacteriana.

Em uma revisão bibliográfica realizada por Jacoby et al. (2017), destacaram-se alguns estudos com a utilização de probióticos, sendo que somente uma parte destes apresentou propriedades benéficas. Os probióticos utilizados foram compostos por Lactobacillus paracasei, L. plantarum, $L$. acidophilus, L. rhamnosus, L. brevis e L. delbrueckii (86,70\%), por Bifidubacterium lactis, B. longum, B. breve, B. bifidum e B. infantis (60\%) e, também, por Pediococcus pentosaceus, Leuconostoc mesenteroides, Saccharomyces boulardii, Enterococcus faecalis, Bulgaricus e Streptococcus thermophilus. Os efeitos encontrados foram: diminuição da proteína C-reativa (PCR), da incidência e da severidade da diarreia, do risco de complicações pós-operatórias, como sepse, ventilação mecânica e vazamento da anastomose, além da recuperação mais rápida da 
função intestinal. Em apenas $21,4 \%$ dos estudos os resultados esperados não foram atingidos. Dentre os estudos descritos nessa revisão podemos citar:

Zhang et al (2012) demonstrou em seu estudo que três cápsulas de 0,21 g de Blongum, Lactobacillus acidophilus e Enterecoccus faecalis, três vezes ao dia, por um período de três dias, têm efeito benéfico na diminuição das complicações infecciosas no pós-operatório, minimizando o crescimento excessivo de Escherichia e. coli no intestino. Além disso, restringem a translocação bacteriana, representando um aumento da imunidade sistêmica e na resposta ao estresse sistêmico.

Para Stephens \& Hewett (2012), em um estudo realizado com probióticos, composto por Streptococcus thermophilus, Bifidobacterium breve, Bifidobacterium Longum, Bifidobacterium infantis, Lactobacillus acidophilus, Lactobacillus casei, Lactobacillus delbrueckii subsp. Bulgaricus e Lactobacillus plantarum, com a dose de 2,5 gramas, três vezes ao dia por quatro semanas, observou-se que não houve efeito benéfico quando utilizado na prevenção ou redução dos sintomas intestinais desordenados do pós-operatório de pacientes com síndrome de ressecamento anterior, sofrendo reversão de ileostomia.

Martinez (2008) realizou um estudo com 196 mulheres, dentre elas 64 saudáveis, 68 com candidíase vulvovaginal e 64 com vaginose bacteriana. As pacientes foram selecionadas e examinadas pelos médicos ginecologistas de um centro de atendimento de Ribeirão Preto - SP. Observou-se a presença e $\mathrm{o}$ aspecto do corrimento vaginal, determinou-se o $\mathrm{pH}$ da vagina e coletaramse três amostras de secreção de cada paciente. As pacientes diagnosticadas com candidíase vulvovaginal e vaginose bacteriana foram tratadas com medicamentos e suplementação de Lactobacillus rhamnosus e L. leuteri. Algumas receberam placebo durante o tratamento. Os grupos foram formados por 30 sujeitos, sendo que em 30 dias as mulheres deveriam refazer os exames. Das 68 mulheres diagnosticadas com candidíase vulvovaginal, apenas 55 foram selecionadas para a análise estatística, pois 13 tiveram amostras negativas para cândida. 29 delas foram tratadas com dose única de fluconazol $(150 \mathrm{mg})$ e com cápsulas diárias de probióticos; as demais receberam o fluconazol e o placebo. No término das quatro semanas de tratamento, pode-se concluir que em $89,7 \%$ dos casos houve cura da candidíase vulvovaginal das mulheres tratadas com fluconazol e probióticos, além de desaparecimento dos sintomas clássicos e das culturas negativas, quando comparadas as outras $65,4 \%$ que receberam fluconazol e placebo.

Carvalho (2007) avaliou o potencial probiótico de 15 amostras de Lactobacillus crispatus e de seis amostras de Lactobacillus johnsonii. Essas espécies prevaleceram em estudo prévio com 16 mulheres saudáveis. Os Lactobacillus crispatus revelaram atividade antagonista contra um maior número de linhagens de cândida. Nesse estudo, foram realizados testes in vitro (teste de antagonismo com linhagens de leveduras de Candida albicans, tropicalis e krusei) e in vivo (utilizando fêmeas de camundongos). As leveduras de cândida foram 
aplicadas nos camundongos por via intravaginal em três etapas. Observou-se diferença estatística apenas quando aplicada a Candida tropicalis; nesse caso, os Lactobacillus não reduziram a infecção, mas diminuíram os níveis populacionais das leveduras. $\mathrm{O}$ estudo concluiu que os dados obtidos indicam provável atividade probiótica, mas necessitam de melhor avaliação de suas propriedades antagonistas e de sua aplicabilidade no tratamento de candidíase vaginal.

Matsubara (2016) avaliou o efeito de bactérias probióticas sobre a Candida albicans, em culturas de macrófagos e biofilmes. Observou-se que os Lactobacillus probióticos são capazes de interferir nos macrófagos para reconhecimento da Candida albicans, reduzindo as citocinas pró-inflamatórias. Concluiu-se que os lactobacillus são capazes de inibir a diferenciação de Candida albicans e o desenvolvimento do seu biofilme, principalmente na fase de colonização inicial da levedura.

Xie et al. (2017) realizou um estudo com mulheres não grávidas, por meio do qual avaliou a eficácia dos probióticos no tratamento da candidíase vulvovaginal. Todos os testes utilizaram probióticos como coadjuvantes para os medicamentos antifúngicos. Conclui-se que, quando comparados ao tratamento normal, o uso de probióticos pode aumentar a cura clínica e fungicida em curto prazo e dos casos recorrentes no prazo de um mês. Não foi verificada eficácia em longo prazo. Para isso, o autor sugere nova pesquisa com maior amostra e acompanhamento, com metodologias padronizadas.

Pericolini et al. (2017) estudou o efeito do Saccharomyces cerevisiae (fermento utilizado para produção de igredientes para nutrição humana) como probiótico influenciador na melhora de candidíase vulvovaginal. Em uma parte da população pesquisada foi administrado por via vaginal a levedura viva do probiótico e em outra parte o fermento inativado. Observou-se que o fermento vivo teve maior eficácia, influenciando positivamente na terapêutica, beneficiando a mucosa vaginal e eliminando a infecção por candidíase.

Davar et al. (2016) comparou em seu estudo a recorrência de candidíase vulvovaginal após o tratamento oral com dose única de fluconazol 150mg e administração de probióticos. A amostra foi de 59 pacientes diagnosticadas com candidíase por seis meses, dividindo-as em dois grupos: 31 mulheres receberam placebo e as outras 28 receberam probiótico. No decorrer dos seis meses, observou-se que, no grupo placebo, onze pacientes adquiriram candidíase de repetição e, naquelas que utilizaram probiótico, somente duas adquiriram. Os estudos demonstram que administrar probióticos com antifúngicos pode ser eficaz para o tratamento de candidíase vulvovaginal e também de repetição.

Hanson (2016) realizou uma revisão sistemática com 20 estudos para determinar a eficácia dos probióticos na prevenção e no tratamento de infecções urogenitais em mulheres adultas. Dentre os estudos analisados, dois eram focados em candidíase vaginal. Pode-se observar que, apesar das recomendações para prática clínica terem sido limitadas, os probióticos foram efetivos tanto no tratamento quanto na prevenção, sendo utilizados como 
alternativos ou coadjuvantes. Os estudos demonstraram também eficácia na candidíase de repetição.

\section{CONSIDERAÇÕES FINAIS}

Nesta revisão de literatura, pode-se observar que poucos estudos não obtiveram benefícios com a suplementação de probióticos para reduzir os sintomas intestinais. Quando relacionados ao tratamento de candidíase vulvaginal, em apenas um dos estudos observou-se que os Lactobacillus não reduziram as infecções causadas por Candida tropicalis, mas ainda assim diminuíram a população inicial das leveduras.

Conclui-se, portanto, que os probióticos apresentam efeitos benéficos no equilíbrio da flora intestinal, aumentam o sistema imune e são coadjuvantes no tratamento para candidíase vaginal e de repetição. Ainda não existem estudos que comprovem sua eficácia quando administrados individualmente; para isso, serão necessárias novas pesquisa in vivo, com maior número de mulheres diagnosticadas com candidíase vulvovaginal.

\section{REFERÊNCIAS}

BALDIM, I.M.; PEREIRA, M.A.; RUFINO, L.R.A.; OLIVEIRA, N.M.S.; FIORINI, J.E. Teste de sensibilidade ao quefir de cepas de Candida sp. isoladas de vulvovaginites. Rev Ciênc Farm Básica Apl., 2012; 33(3):379-383. Disponível em: <http:/ /serv-bib. fcfar.unesp.br/seer/index.php/Cien_Farm/article/viewFile/2078/1263>. Acesso em 26 de out. de 2017.

BARBOSA, Claudia.; FERNANDES, Gustavo; QUINTAS, Carolina; TEIXEIRA, Ester M.; NEVES, João Pedro. Derivados azólicos no tratamento da candidíase vulvovaginal não complicada. Acta Obstet Ginecol Port 2012;6(3):118-123. Disponível em: <http://www.fspog.com/fotos/editor2/1_ficheiro_607.pdf $>$. Acesso em 15 de nov. 2017.

CARVALHO, Fabio P. Avaliação in vitro e in vivo do potencial probiótico de linhagens de Lactobacillus provenientes do canal vaginal de mulheres saudáveis, contra Candida spp. Instituto de Ciências Biológicas da UFMG, 2007. Belo Horizonte. Disponível em: <http:/ /www.bibliotecadigital.ufmg.br/dspace/bitstream/ handle/1843/BUOS-8E5EKR/fabio.pdf?sequence=1>. Acesso em 16 de nov. 2017.

DAVAR, Robab; NOKHOSTIN, Fahimeh; EFTEKHAR, Maryam; SEKHAVAT, Leili; ZADEH, Mohammad B.; SHAMSI, Farimah. Comparing the Recurrence of Vulvovaginal Candidiasis in Patients Undergoing Prophylactic Treatment with Probiotic and Placebo During the 6 Months. Probiotics \& Antimicro. Prot. DOI: 10.1007/s12602-016-9218-x. Disponível em: <file:///C:/Users/user/Downloads/ estudo\%203\%20(1).pdf>. Acesso em 22 de jan. 2018. 
FARJADO, A.C.S. Caracterização do microbioma humano. Instituto Superior de Ciências da Saúde Egas Moniz. Mestrado Integrado em Ciências Farmacêuticas. 2015. Disponível em: <https:/ / comum.rcaap.pt/bitstream/10400.26/10975/1/ Fajardo\%2C\%20Anabela\%20Cristina\%20da\%20Silva.pdf>. Acesso em 06 de jul. 2017.

FERREIRA, Geysa S. Disbiose intestinal: aplicabilidade dos prebióticos e dos probióticos na recuperação e manutenção da microbiota intestinal. Centro Universitário Luterano de Palmas. Palmas- TO, 2014. Disponível em: <file:/ / C:/ Users/user/Downloads/document55e9f4b59e0bd\%20(1).pdf>. Acesso em 09 de jan. 2018.

FEUERSCHUETTE, Otto Henrique M.; SILVEIRA, Sheila K.; FEUERSCHUETTE, Irmoto; CORREAA, Tiago; GRANDO, Leisa; TREPANI, Alberto. Candidíase vaginal recorrente: manejo clínico. FEMINA | Fevereiro 2010 | vol $38 \mid \mathrm{n}^{\circ} 2$. Disponível em: <http:/ / files.bvs.br/upload/S/0100-7254/2010/v38n1/a005.pdf >. Acesso em 16 de nov. 2017.

FONSECA, Fernanda C.P.; COSTA, Célia L. Influência da nutrição sobre o sistema imune intestinal. CERES; 2010; 5(3); 163-174. Disponível em: <http:/ / www.epublicacoes.uerj.br/index.php/ceres/article/view/1946/1508>. Acesso em 07 de jul. 2017.

HANSON, Lisa; VANDEVUSSE, Leona; JERMÉ, Martha; ABAD, Cybéle L.; SAFDAR, Nasia. Probiotics for Treatment and Prevention of Urogenital Infections in Women: A Systematic Review. Journal of Midwifery \& Women's Health. Volume 61, No. 3, May/June 2016. DOI:10.1111/jmwh.12472. Disponível em: <file:/ / C:/Users/user/ Downloads/estudo\%204\%20(1).pdf>. Acesso em 22 de jan. 2018.

HOLANDA, Antonio Arildo Reginaldo.; FERNANDES, Ana Cristina S.; BEZERRA, Christiane M.; FERREIRA, Maria Angela F.; HOLANDA, Manoel Reginal da R.; HOLANDA, Julita de C. P.; MILAN, Eveline P. Candidíase vulvovaginal: sintomatologia, fatores de risco e colonização anal concomitante. Rev Bras Ginecol Obstet. 2007; 29(1):3-9. Disponível em: <http:/ / www.scielo.br/pdf/rbgo/v29n1/ a02v29n1.pdf>. Acesso em 15 de nov. 2017.

JACOBY, Jésica T.; GUZZON, Simone; ROESCH, Luiz Fernando W.; MENDES, Roberta H. Uso de pré, pró e simbióticos como coadjuvantes no tratamento do câncer colorretal. Clin Biomed Res. 2017;37(3):232-246. Disponível em: <http:/ / seer. ufrgs.br/index.php/hcpa/article/view/72730/pdf_1>. Acesso em 26 de out. 2017.

MARTINEZ, R.C.R. Efeito da utilização de culturas láticas probióticas na microbiota vaginal de pacientes acometidas por infecções bacterianas e fúngicas. Faculdade de Ciências Farmacêuticas de Ribeirão Preto. Ribeirão Preto, 2008. Disponível em: <file:/ / C:/Users/user/Downloads/Tese\%20(1).pdf>. Acesso em 16 de nov. de 2017.

MATSUBARA, V.H. Efeito de bactérias probióticas sobre Candida albicans: ensaios e cultura de macrófagos e de biofilme. Faculdade de Odontologia da Universidade de São Paulo. São Paulo, 2016. Disponível em: <file:/ / C:/Users/user/Downloads / VictorHaruoMatsubaraVersaoOriginal\%20(1).pdf>. Acesso em 16 de nov. 2017. 
MELO, B.J.C.; NASCIMENTO, E.E.F.; SOARES, S.J.T.; SOUZA, L.T.S.;

PARAHYM, A.M.R.C. Candidíase vulvovaginal: Revisão de literatura.

2016. Disponível em: <https:/ / even3.azureedge.net/processos /

ARTIGOCANDIDIASEVULVOVAGINALREVISAODALITERATURA.

c566b7c4d3aa483397d7.pdf>. Acesso em 15 de nov. 2017.

MORAES, Fernanda P.; COLLA, Luciane M. Alimentos funcionais e nutracêuticos: definições, legislação e benefícios à saúde. Revista Eletrônica de Farmácia Vol 3(2), 109-122, 2006. Disponível em: <https:/ / www.revistas.ufg.br/REF/article / viewFile/2082/2024>. Acesso em 24 de out. 2017.

PAIXÃO, L.A.; CASTRO, F.F.S. A colonização da microbiota intestinal e sua influência na saúde do hospedeiro. Universitas: Ciências da Saúde, Brasília, v. 14, n. 1, p. 85-96, jan./jun. 2016. Disponível em: <https://publicacoesacademicas.uniceub. br/cienciasaude/article/viewFile/3629/3073. Acesso em 06 de jul. 2017.

PEREIRA, I.G.; FERRAZ, I.A.R. Suplementação de glutamina no tratamento de doenças associadas à disbiose intestinal. Revista Brasileira de Saúde Funcional. 2017. Disponível em: <file:/ / C:/Users/user/Downloads/830-3130-1-PB\%20(1).pdf>. Acesso em 06 de jul. 2017.

PERICOLINI, Eva; GABRIELLI, Elena; BALLET, Nathalie; SABBATINI, Samuele; ROSELLETTI, Elena; DECHERF, Amelia C.; PÉLERIN, Fanny; LUCIANO, Eugenio; PERITO, Stefano; JÜSTEN, Petter; VECCHIARELLI, Anna. (2017) Therapeutic activity of a Saccharomyces cerevisiae-based probiotic and inactivated whole yeast on vaginal candidiasis, Virulence, 8:1, 74-90, DOI: 10.1080/21505594.2016.1213937. Disponível em:<https://doi.org/10.1080/21505594.2016.1213937>. Acesso em 22 de jan. 2018.

RODRIGUES, Marcio T.; GONÇALVES, Ana Carolina; ALVIM, Maria Carolina T.; CASTELLANO FILHO, Didier S.; ZIMMERMMANN, Juliana B.; SILVA, Vânia Lucia; DINIZ, Cláudio G. Associação entre cultura de secreção vaginal, características sociodemográficas e manifestações clínicas de pacientes com diagnóstico de candidíase vulvovaginal. Rev Bras Ginecol Obstet. 2013; 35(12):554-61. Disponível em:< http:/ / www.scielo.br/pdf/rbgo/v35n12/05.pdf>. Acesso em 16 de nov. 2017.

STEPHENS, J.H; HEWETT, P.J. Clinical trial assessing VSL\#3 for the treatment of anterior resection syndrome. ANZ J Surg. 2012;82(6):420-7. PMid:22571474.

Disponível em:<http:/ /dx.doi.org/10.1111/j.1445- 2197.2012.06082.x.>. Acesso em 26 de out. 2017.

VANDENPLAS, Yvan; HUYS, Geert; DAUBE Georges. Probiotics: na update. J Pediatr (Rio J). 2015;91(1):6---21. Disponível em: <http:/ / www.scielo.br/pdf/jped/ v91n1/pt_0021-7557-jped-91-01-00006.pdf>. Acesso em 26 de out. 2017.

XIE, Huan Y.; FENG, Dan; WEI, Dong M.; MEI, Ling; CHEN, Hui; WANG, Xun; FANG, Fang. Probiotics for vulvovaginal candidiasis in non-pregnant women. Cochrane Database of Systematic Reviews 2017, Issue 11. Art. No.: CD010496. 
DOI: 10.1002/14651858.CD010496.pub2. Disponível em: <file:/ / C:/Users/user/ Downloads/estudo\%201.pdf>. Acesso em 22 de jan. 2018.

ZHANG, J.W.; DU, P.; GAO, J.; YANG, B.R.; FANG, W.J.; YING, C.M. Preoperative probiotics decrease postoperative infectious complications of colorectal cancer. Am J Med Sci. 2012;343(3):199-205. PMid:22197980. Disponível em: <https:/ / www.ncbi.nlm. nih.gov/pubmed/22197980>. Acesso em 26 de out. 2017. 